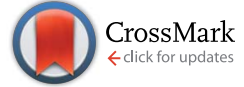

Cite this: RSC Adv., 2017, 7, 12113

\title{
Sequential crystallization of high and low melting waxes to improve oil structuring in wax-based oleogels $\dagger$
}

\author{
Iris Tavernier, $\star^{\star a}$ Chi Diem Doan, $\stackrel{+}{\text { ab }}^{\text {Da }}$ Davy Van de Walle, ${ }^{a}$ Sabine Danthine, ${ }^{c}$ \\ Tom Rimaux ${ }^{d}$ and Koen Dewettinck ${ }^{a}$
}

The main objective of this study was to investigate the effect of combining a high-melting wax (sunflower wax or rice bran wax) with a low-melting wax (berry wax or BEW) on the crystallization and gelation behavior of the corresponding wax-based oleogels in rice bran oil (RBO). Sunflower wax (SW) and rice bran wax (RBW) have a similar chemical composition but a very different crystallization behavior. Therefore, SW and RBW were also combined in a wax-based oleogel to examine the occurrence of co-crystallization and/or crystal coexistence. The thermal and gelation behavior of the binary blends in rice bran oil $(5 \% \mathrm{w} / \mathrm{w}$ total concentration of wax) were investigated with differential scanning calorimetry (DSC) and rheological measurements, showing sequential crystallization and gelation for the SW/RBW : BEW wax-based oleogels and simultaneous crystallization and gelation for the SW : RBW wax-based oleogels. Oscillatory shear and hardness measurements revealed the reinforcement of the high-melting wax crystal network with the addition of the low-melting berry wax. X-ray diffraction (XRD) measurements in both the long-spacing and the short-spacing region showed the occurrence of crystal co-existence, rather than co-crystallization, which was confirmed with polarized light microscopy (PLM). Two different crystal morphologies could be discerned for all three combinations (RBW : SW, SW : BEW and RBW : BEW). We hypothesized that the improved rheological properties could be attributed to sintering, a process in which the low-melting BEW crystals form solid bonds between the crystals of the high-melting waxes. As such, a cohesive network is formed, resulting in an increased hardness of the bi-wax-based oleogels. This research provides the opportunity to minimize the amount of wax necessary in creating semi-solid materials with a wide variety in rheological and textural properties.

Received 1st December 2016 Accepted 13th February 2017

DOI: $10.1039 / c 6 r a 27650 d$

rsc.li/rsc-advances

\section{Introduction}

Traditionally, the lipid phase in food products is structured with crystalline triacylglycerol (TAG) hardstocks. These hardstocks mainly consist of saturated fatty acids (SaFA) or even trans fatty acids (tFA), which have been shown to exert negative effects on the risk markers for cardiovascular diseases., ${ }^{1,2}$ Therefore, SaFA and tFA should be reduced (for SaFA) or even completely eliminated (for tFA) and replaced with unsaturated fats to improve the nutritional profile of our diet. ${ }^{3}$ Alternative

\footnotetext{
${ }^{a}$ Laboratory of Food Technology and Engineering, Department of Food Safety and Food Quality, Faculty of Bioscience Engineering, Ghent University, Coupure Links 653, 9000 Gent, Belgium. E-mail: Iris.Tavernier@UGent.be

${ }^{b}$ Department of Food Technology, Cantho University, Cantho, Vietnam 'Department of Food Science, Université de Liège, Gembloux, Belgium

${ }^{d}$ Vandemoortele R\&D Centre, Prins Albertlaan 79, 8870 Izegem, Belgium

$\dagger$ Electronic supplementary information (ESI) available. See DOI: $10.1039 /$ c6ra27650d

$\$$ Both authors contributed equally to this work.
}

structuring methods are intensely researched to anticipate this consumer demand.

Oleogelation has attracted attention as a possible oilstructuring method. Oleogels are soft matter systems with liquid oil as the continuous phase (usually above $90 \% \mathrm{w} / \mathrm{w}$ ) that is physically entrapped in a network of self-assembled gelator molecules. ${ }^{4}$ According to Co \& Marangoni (2012), the ideal fat structurant should ${ }^{1}$ meet stringent regulatory requirements for use in food, ${ }^{2}$ be affordable and readily available, ${ }^{3}$ be able to structure lipids at relatively low concentrations,${ }^{4}$ exhibit similar physical properties as the fat it is intended to replace $a^{5} d^{5}$ be versatile.

Among various studied oleogelators, waxes appear to be the most promising ones. ${ }^{6}$ They meet the aforementioned requirements and are able to crystallize in a well-defined threedimensional network with a high oil-binding capacity. ${ }^{7}$ Furthermore, they form thermo-reversible oleogels at very low concentrations (as low as $0.5 \% \mathrm{w} / \mathrm{w}$ ) and they can stabilize water-in-oil emulsions. ${ }^{6,8,9}$ Waxes have a multicomponent chemical nature, existing of wax esters (WEs), hydrocarbons (HCs), fatty alcohols (FALs), fatty acids (FAs), ketones, mono-, di-, triacylglycerols and 
sterol esters. ${ }^{6,8}$ Different waxes such as rice bran wax (RBW), sunflower wax (SW) and berry wax (BEW), have a significantly different chemical composition. RBW and SW consist mainly of long-chain aliphatic esters $\left(\mathrm{C}_{36}-\mathrm{C}_{58}\right)$, but differ in $\mathrm{C}_{24}$ free fatty acid content while BEW is primarily comprised of short chain fatty acids $\left(\mathrm{C}_{16}-\mathrm{C}_{18}\right)$, with a prominent amount being bound on the glycerol backbones as diacylglycerols (DAGs) and triacylglycerols (TAGs). ${ }^{\mathbf{8 1 0}-13}$ These chemical differences will result in different crystallization behavior, and therefore also in different functional properties. RBW and SW are high-melting waxes due to the presence of a high amount of long-chain WEs but RBW exhibits big dendritic crystals and forms weak oleogels in rice bran oil while SW reveals platelet crystals which connect into a dense and strong network. The difference can be explained by the minor free fatty acid fraction in RBW which forms the dendritic molecules that interfere with the network development of platelet crystals. ${ }^{12} \mathrm{BEW}$ is a low-melting wax with strong gelling properties due to the presence of tiny platelet crystals in the crystal network. ${ }^{\mathbf{1 4}}$ Possibly, combining different waxes can open up new opportunities for designing tailor-made wax-based materials with specific properties for various food applications.

Several studies have researched the crystallization of waxes in liquid oil: candelilla wax in safflower oil, ${ }^{15}$ rice bran wax in olive oil, ${ }^{16}$ beeswax and sunflower wax in olive oil, ${ }^{17}$ sunflower wax in milk fat, ${ }^{18}$ several waxes in rice bran oil, ${ }^{\mathbf{1 4}}$ etc. Jana and Martini (2016) studied the phase behavior of binary blends of four different neat waxes to determine the total amount of material that can be crystallized and to characterize the types of crystalline networks formed in terms of crystal morphology. ${ }^{19}$ Recently, they also published a paper on the use of binary blends of waxes in soybean oil, but only high-melting waxes (beeswax, sunflower wax and rice bran wax) were combined and no enhanced structuring effect was obtained. ${ }^{20}$

The objective of this study was to combine two waxes to enhance the resistance to small and large deformations of the respective oleogel. The oleogels were prepared with rice bran oil because of its relatively low content of saturated fatty acids $(\approx 20 \% \mathrm{w} / \mathrm{w})$ and high amounts of nutritional substances like oryzanol and antioxidants. ${ }^{21}$ We hypothesized that a stronger wax-based oleogel could be achieved by combining a high and a low melting wax. Upon crystallizing, the high melting wax crystallizes first and forms the initial network. Subsequently, the low-melting wax crystallizes, which is possibly positively influenced by the prior presence of the high-melting wax crystals. To test this hypothesis, SW and RBW (high-melting waxes) were combined with BEW (a low-melting wax). SW was also combined with RBW because they have a nearly similar crystallization temperatures $\left(57^{\circ} \mathrm{C}\right.$ for RBW and $62{ }^{\circ} \mathrm{C}$ for SW) and a similar chemical composition (long chain WEs), which could result in interesting crystalline structures.

The thermal and gelation behavior of the binary blends in rice bran oil ( $5 \% \mathrm{w} / \mathrm{w}$ total concentration of wax) were investigated with differential scanning calorimetry (DSC) and temperature ramp measurements. The response of the bi-waxbased oleogels to small and large deformations was determined with oscillatory shear measurements and hardness measurements. To structurally explain the results of the hardness measurements and to test the formation of mixed molecular compounds or new polymorphs, X-ray diffraction measurements were combined with solid fat content measurements. Polarized microscopy allowed the identification of the crystal morphologies occurring in the mixed systems.

\section{Experimental}

\section{Materials}

Rice bran oil (RBO, onset and peak melting temperatures: $T_{\mathrm{m} \text {,onset }}=-73.95^{\circ} \mathrm{C}, T_{\mathrm{m} \text {,peak }}=-11.14{ }^{\circ} \mathrm{C}$ ) (Suriny brand, Surin Bran Oil Company, Thailand) was purchased from a local supermarket in Ghent (Belgium). The fatty acid composition of RBO was the following: oleic acid $(44.2 \% \mathrm{w} / \mathrm{w})$, linoleic acid $(30.0 \% \mathrm{w} / \mathrm{w})$, palmitic acid $(19.9 \% \mathrm{w} / \mathrm{w})$, stearic acid $(2.1 \% \mathrm{w} / \mathrm{w})$ and arachidic acid $(1.1 \% \mathrm{w} / \mathrm{w})$. Sunflower wax (SW, $96 \%$ of wax esters - WEs, $3 \%$ of free fatty acids - FFAs, $0.3 \%$ of fatty alcohols - FALs and $0.2 \%$ of hydrocarbons - HCs) and rice bran wax (RBW, $93 \%$ of WEs, $6 \%$ of FFAs, $0.2 \%$ of FALs and $0.3 \%$ of HCs) were kindly supplied by Koster Keunen Holland BV Co (Bladel, The Netherlands). Berry wax - extracted from the fruits of the Rhus verniciflua tree of Asia (BEW, 95\% of FAs and most of them bound on the glycerol backbones, $4 \%$ of FALs and 1\% of WEs) was donated by Kalhwax GmbH \& Co. KG (Trittau, Germany). The chemical composition of the waxes was determined inhouse using HPLC-ELSD. Details on the experimental set-up and compositional analysis have been reported previously. ${ }^{12}$

\section{Preparation of the oleogels}

Two high-melting waxes (SW and RBW) and one low-melting wax (BEW) were combined in different ratios from 0 to $100 \%$ in $10 \%$ increments to create bi-gelator systems (RBW: SW, RBW : BEW and SW:BEW). To prepare the oleogel, each combination was dispersed in RBO at a constant total concentration of $5.0 \% \mathrm{w} / \mathrm{w}$ in $\mathrm{RBO}$. This concentration was used because it is the minimum gelling concentration of RBW in RBO. ${ }^{14}$ As reference, also mono-wax based oleogels were prepared in the concentrations of $4,2.5$ and $1 \% \mathrm{w} / \mathrm{w}$. All the samples were heated at $90{ }^{\circ} \mathrm{C}$ under mild agitation $(200 \mathrm{rpm})$ using a magnetic stirrer (Model EM300T, Labotech Inc., Germany) for 10-15 min until clear oily solutions were obtained. These solutions were subsequently statically cooled to $5{ }^{\circ} \mathrm{C}$ at a cooling rate estimated at $5{ }^{\circ} \mathrm{C} \min ^{-1}$ and stored at $5{ }^{\circ} \mathrm{C}$ overnight in a thermal cabinet for further experiments.

\section{Thermal behavior}

The thermal profiles of bi-waxes in RBO were obtained with a Q1000 DSC (TA Instruments, New Castle, Delaware, USA) equipped with a refrigerated cooling system. Nitrogen was used as purge gas. The cell constant and temperature were set with indium (TA Instruments). An additional temperature calibration was done using azobenzene (Sigma-Aldrich, Bornem, Belgium) and undecane (Acros Organics, Geel, Belgium). The sample (weighing between 6.0 and $8.0 \mathrm{mg}$ per cup) was placed inside an aluminum pan and sealed with an aluminum lid (TA Instruments). The samples were subjected to 2 cycles. In the 
first cycle, the sample was equilibrated at $90{ }^{\circ} \mathrm{C}$ in $10 \mathrm{~min}$ and cooled to $0{ }^{\circ} \mathrm{C}$ at a cooling rate $5{ }^{\circ} \mathrm{C} \min ^{-1}$ to determine the crystallization temperatures. In the second cycle, the samples were first equilibrated at $90{ }^{\circ} \mathrm{C}$ in $10 \mathrm{~min}$ to erase all the crystal history, followed by cooling to $5{ }^{\circ} \mathrm{C}$ at a rate of $5{ }^{\circ} \mathrm{C} \mathrm{min}{ }^{-1}$, kept isothermally at $5{ }^{\circ} \mathrm{C}$ in $10 \mathrm{~min}$ before being re-heated to $90{ }^{\circ} \mathrm{C}$ at a rate of $5{ }^{\circ} \mathrm{C} \mathrm{min}^{-1}$ to determine the melting profile for phase diagram. The characteristic parameters of thermal curves $\left(T_{\mathrm{c}, \mathrm{on}}\right.$, $\left.T_{\text {c,peak }}, T_{\mathrm{m} \text {,on }}, T_{\mathrm{m} \text {,peak }}, T_{\mathrm{m} \text {,off }}\right)$ were acquired using the software TA Universal Analysis provided by the instrument supplier. The phase diagram was built using the onset $\left(T_{\mathrm{m}, \mathrm{on}}\right)$ and offset melting temperatures $\left(T_{\mathrm{m} \text {,off }}\right)$, obtained by determining the running integral over the melting peak. The running integral was expressed in area percentage, indicating the fraction of the material that was melted, as a function of temperature. $T_{\mathrm{m} \text {,on }}$ and $T_{\mathrm{m} \text {,off }}$ were defined as the temperatures at which the area percentage of the melting peak was $1 \%$ (the material started melting), respectively $99 \%$ (the material was almost completely melted). The average $T_{\mathrm{m} \text {,on }}$ and $T_{\mathrm{m} \text {,off }}$ were calculated from 3 repetitions. $T_{\mathrm{m} \text {,on }}$ and $T_{\mathrm{m} \text {,off }}$ values were plotted as a function of the composition of the bi-gelator system in RBO.

\section{Rheological behavior}

All the rheological measurements were carried out using an advanced rheometer AR2000ex (TA Instruments, New Castle, USA) equipped with a Peltier system and water bath (Julabo, Seelbach, Germany) for temperature control. The starch pasting cell (shear rate factor $=4.500 \mathrm{~s}^{-1}$, shear stress factor $=48600$ $\mathrm{m}^{-3}$, gap $=5500 \mu \mathrm{m}$ ) was utilized to record the gelling points during cooling from 90 to $5{ }^{\circ} \mathrm{C}$ at a cooling rate of $5{ }^{\circ} \mathrm{C} \mathrm{min}^{-1}$. The oleogels were then kept at $5{ }^{\circ} \mathrm{C}$ for $30 \mathrm{~min}$ to investigate the isothermal change in gelling properties of wax-based oleogels. Parallel plate (cross-hatched; diameter, $\phi=40.0 \mathrm{~mm}$; gap = $1000 \mu \mathrm{m})$ geometry was used to observe the linear visco-elastic region (LVR) and the time-dependent deformation behavior (frequency sweep) of the wax-based oleogels. The LVR was determined by logarithmically increasing the oscillation stress from 0.01 to $1000 \mathrm{~Pa}$ at a frequency of $1.0 \mathrm{~Hz}$. Frequency sweeps (0.01-100 Hz) were subsequently performed at $5{ }^{\circ} \mathrm{C}$ and a stress value within LVR.

\section{Texture analysis}

The hot wax-based solutions were placed in $5 \mathrm{~cm} \times 3 \mathrm{~cm}$ plastic cups, cooled to $5{ }^{\circ} \mathrm{C}$ at a cooling rate estimated at $5{ }^{\circ} \mathrm{C} \mathrm{min}^{-1}$ and stored at $5{ }^{\circ} \mathrm{C}$ in a thermostatic cabinet in 7 days before analysis. An $11 \mathrm{~mm}$ diameter cylindrical plastic probe penetrated the sample to a depth of $10 \mathrm{~mm}$ at a rate of $10 \mathrm{~mm} \mathrm{~min} \mathrm{~m}^{-1}$ with a $0.1 \mathrm{~N}$ trigger value at $5{ }^{\circ} \mathrm{C}$. The firmness was determined as the force required for penetration using a 5942 Instron TA 500 Texture Analyzer (Lloyd Instrument, Bognor Regis, West Sussex, UK).

\section{Solid fat content}

The solid fat content of three ratios (80:20,50:50 and $20: 80)$ of each wax combination were was measured at $5{ }^{\circ} \mathrm{C}$ using Maran Ultra $23 \mathrm{MHz}$ pulsed Nuclear Magnetic Resonance
(pNMR) equipment (Oxford Instruments, Abingdon, UK). The NMR-tubes were filled with $4 \mathrm{~mL}$ of wax blends. Prior to analysis, the samples were conditioned in the oven at $85{ }^{\circ} \mathrm{C}$ to erase the crystal memory. Next, they were transferred into the cooling water bath at $5{ }^{\circ} \mathrm{C}$ for $24 \mathrm{~h}$ after which the FID signal was recorded. Using calibration standards (0\%, 29.4\% and 70.6\% SFC), SFC values were derived.

\section{Powder X-ray diffraction spectroscopy}

The occurrence of mixed crystals and/or co-existing crystals in the binary blends (RBW : SW, RBW : BEW and SW : BEW) in RBO was investigated by XRD using a Bruker D8-Advanced Diffractometer (Bruker, Germany) $\left(l_{\mathrm{Cu}}=1.54178 \AA\right.$, $40 \mathrm{kV}$, and $30 \mathrm{~mA}$ ), equipped with an Anton Paar temperature control system composed of a TTK 450 low-temperature chamber connected to a waterbath (Lauda) and heating device (TCU 110 Temperature Control Unit) (Anton Paar, Graz, Austria). The samples were heated at $90{ }^{\circ} \mathrm{C}$ for 10 minutes and subsequently cooled to $5{ }^{\circ} \mathrm{C}$ at a cooling rate of $5{ }^{\circ} \mathrm{C} \mathrm{min}^{-1}$. The short-spacing runs were recorded at $5{ }^{\circ} \mathrm{C}$ using a Vantec- 1 detector (Bruker, Germany). $D$-Values were directly calculated by Diffract.Suite Eva software.

\section{Cryo-scanning electron microscopy}

To visualize the crystal network within the wax-based oleogels, the oleogel samples were first partly de-oiled with ethanol to remove the surface oil and the oil bound within the crystal network. A known quantity of oleogel was mixed with ethanol and mildly stirred, resulting in the partial collapse of the gel structure and the subsequent sedimentation of the crystalline fraction. The suspension was stored overnight at $5{ }^{\circ} \mathrm{C}$. The supernatant liquid was decanted and the crystalline sediment was collected after filtration through a filter paper. This sediment was then placed on the sample holder, which was plungefrozen in liquid nitrogen and transferred into the cryopreparation chamber (PP3010T Cryo-SEM Preparation System, Quorum Technologies, UK). The frozen sample was freezefractured to observe the internal structure. Subsequently, the sample was sputter-coated with Pt and examined in a JEOL JSM 7100F SEM (JEOL Ltd., Tokyo, Japan).

\section{Polarized light microscopy}

Three ratios (80:20, $50: 50$ and $20: 80$ ) of each wax combination were chosen to investigate their crystallization behavior under polarized light microscopy (PLM). The crystal morphology of the wax-based oleogels was compared at the same concentration of $5.0 \% \mathrm{w} / \mathrm{w}$ bi-wax based oleogels in RBO. After placing the sample on a glass microscope slide and covering it with a slit, the samples were equilibrated at $90{ }^{\circ} \mathrm{C}$ and cooled to $5{ }^{\circ} \mathrm{C}$ at a cooling rate $5{ }^{\circ} \mathrm{C} \min ^{-1}$ on a hot stage connected to a Linkam T95 System Controller (Linkam Scientific Instrument Ltd, Surrey, UK). The microstructure of the wax crystals was observed during cooling to confirm the sequential crystallization and/or co-existence of different crystalline particles. The images were captured under polarized light using 
a Leica DM2500 microscope (Wetzlar, Germany) equipped with a color camera Leica MC170 HD.

\section{Statistical analysis}

The experimental data are expressed as means \pm standard deviation of three repetitions and were analyzed using one factor analysis of variance (ANOVA). The equality of variances were verified using Levene's test prior to usage of Tukey's test to compare the mean values at $p<0.05$ significance level.

\section{Results and discussion}

\section{Phase behavior of the combined waxes in rice bran oil}

Fig. 1 shows the DSC melting profiles and the pseudo-phase diagrams of the three bi-wax-based oleogels researched in this study. The phase diagrams are considered to be pseudo-phase diagrams because waxes are not pure components but have a multicomponent chemical nature, existing out of both polar and apolar components with the major components being hydrocarbons, wax esters, fatty acids, and fatty alcohols..$^{10,12,22}$ The phase diagrams were based upon the respective melting curves (Fig. 1, left) and the characteristic temperatures derived from these curves ( $T_{\text {on }}$ and $T_{\text {off }}$ ). The onset and offset temperature were determined using the running integral, indicating the fraction of the material that was melted, as a function of temperature. This method of determining $T_{\text {on }}$ and $T_{\text {off }}$ is significantly more accurate than using extrapolation from the steep section of the endotherm. For the mixture RBW : SW, which revealed only one melting peak, the onset $\left(T_{\mathrm{m}, \mathrm{on}}\right)$ and the offset temperature $\left(T_{\mathrm{m}, \text { off }}\right)$ were used to create the diagram. For systems with two melting peaks in the melting curves (BEW : SW and BEW : RBW), the diagrams were constructed by plotting the $T_{\mathrm{m}, \text { on }}$ and $T_{\mathrm{m} \text {,off }}$ of the high melting peak ( $\mathrm{SW}$ or RBW) and the $T_{\mathrm{m}, \text { on }}$ and $T_{\mathrm{m} \text {,off }}$ of the low melting peak (BEW). In this way, four lines were formed for the combinations with BEW and two lines for the RBW : SW combination. The top line (-O-) is the liquidus line above which all the material is liquid and the bottom line is the solidus line (- $\mathbf{\nabla}$-), below which all the material is solid. For the combinations with BEW, two additional lines are visible, to indicate the temperatures at which the BEW is melted for $99 \%(-\Delta-)$ and at which temperature the high melting wax (RBW or SW) starts to melt (- $\bullet-)$.

The melting profiles of $5 \% \mathrm{w} / \mathrm{w} \mathrm{RBW}, 5 \% \mathrm{w} / \mathrm{w}$ SW and $5 \%$ $\mathrm{w} / \mathrm{w}$ BEW display one melting peak at respectively $68.1 \pm 0.1{ }^{\circ} \mathrm{C}$, $63.8 \pm 0.2{ }^{\circ} \mathrm{C}$ and $20.5 \pm 1.2{ }^{\circ} \mathrm{C}$. The presence of only one melting peak, confirms the mono-component nature of RBW, SW and BEW. SW and RBW exist mainly out of wax esters, ${ }^{8}$ while BEW is primarily composed of fatty acids. ${ }^{12}$

Upon combining RBW and SW, the single peak observed indicates simultaneous crystallization of two different crystal types (co-existence) or co-crystallization (one type of mixed crystal) or both. The formation of mixed crystals possibly occurred because of the similar chemical composition of RBW and SW. According to Doan et al. (2017) the only difference in chemical composition between RBW and SW is the presence of a higher amount of $\mathrm{C}_{24}$ fatty acid moiety in the wax esters in RBW.

Jana and Martini (2016) have set-up a pseudo-phase diagram for the mixture of pure RBW and SW and our results are congruent with theirs: a continuous solid solution is formed where both waxes are completely soluble over the entire range of concentrations. The higher melting temperature they measured $\left(75.1^{\circ} \mathrm{C}\right.$ for SW and $80.9{ }^{\circ} \mathrm{C}$ for RBW) can be explained by the dilution effect since the melting temperature of the neat waxes will always be higher than that of the wax-based oleogels considered in this paper.

The melting profiles of the BEW : RBW and BEW : SW combinations were similar. Initially the low-melting BEW melted (in the $\mathrm{C}_{\mathrm{SW} / \mathrm{RBW}} / \mathrm{C}_{\mathrm{BEW}} / \mathrm{L}_{\mathrm{BEW}}$ zone), after which also the highmelting wax melted (RBW and SW) at higher temperatures (in the $\mathrm{C}_{\mathrm{SW} / \mathrm{RBW}} / \mathrm{L}_{\mathrm{BEW}} / \mathrm{L}_{\mathrm{RBW} / \mathrm{SW}}$ zone). The phase diagrams reveal monotectic phase behavior with transitions from crystal ${ }_{\text {low }}$ melting/crystal ${ }_{\text {high melting to liquid/crystal to liquid during }}$ melting. ${ }^{23}$ From Table $\mathrm{S} 1 \uparrow$ it can be deduced that the presence of RBW or SW during melting does not influence the melting behavior of BEW. The peak melting temperatures of the monoBEW-based oleogels at the same concentrations $(4 \% \mathrm{w} / \mathrm{w}$ and $2.5 \% \mathrm{w} / \mathrm{w}$ ) are not different to those of the BEW peak when combined with SW or RBW.

The sequential crystallization and gelation of the high- and low-melting waxes were investigated rheologically by measuring the $G^{\prime}$ and $G^{\prime \prime}$ during cooling the sample from $90{ }^{\circ} \mathrm{C}$ to $5{ }^{\circ} \mathrm{C}$ at a rate of $5{ }^{\circ} \mathrm{C} \mathrm{min}^{-1}$ (Fig. 2, left) and subsequent ageing of the samples at $5{ }^{\circ} \mathrm{C}$ during $30 \mathrm{~min}$ (Fig. 2, right). At this cooling rate, the gelling points of $5 \% \mathrm{w} / \mathrm{w}$ RBW, SW and BEW in RBO are respectively $33.0 \pm 5.5{ }^{\circ} \mathrm{C}, 47.1 \pm 0.9{ }^{\circ} \mathrm{C}$ and $7.1 \pm 0.3{ }^{\circ} \mathrm{C}$. The peak crystallization temperatures can be found in Table S1. $\dagger$ There is a prominent delay in gelation for all the oleogels but especially for RBW oleogel, since there is a big difference between $T_{\text {gel }}$ and $T_{\mathrm{c} \text {,peak }}$ of the RBW oleogels $\left(T_{\mathrm{c}, \text { peak,RBW }}=53.71\right.$ $\left.\pm 0.46^{\circ} \mathrm{C}\right)$.

For the SW : RBW combination, one unique gelling point was observed. At the end of the cooling period, the oleogels with a high proportion of SW presented a stronger gelled network which was deduced from the higher elastic modulus $G^{\prime}$. A higher proportion of RBW resulted in less strong gelling behavior, which is in accordance with results obtained by Doan et al. (2015) who found that RBW gelled in RBO from $5 \% \mathrm{w} / \mathrm{w}$ onwards. Yet, Dassanayake, et al. (2009); Hwang, et al. (2012) and Jana \& Martini (2016b) did report on good gelling behavior of RBW in salad oil, olive oil, tea seed oil, sunflower oil and soybean oil. ${ }^{\mathbf{8} 16,24}$ In the research done by Hwang et al. (2012), one of the three RBW also showed bad gelation properties which was explained by a difference in exact composition which strongly influenced its gelation property. ${ }^{8}$ On the other hand, also the type of solvent strongly influences the gelation. Co and Marangoni (2012) stated that an efficient gelator needs to balance its solubility and insolubility in a solvent to achieve proper gelator-gelator and gelator-solvent interactions. ${ }^{5}$ Doan et al. (2015) therefore assumed that RBW has a reasonably high solubility in RBO and, accordingly, acts as a bad oleogelator. ${ }^{14}$ To test the latter hypothesis, the samples were prepared in 
(a)
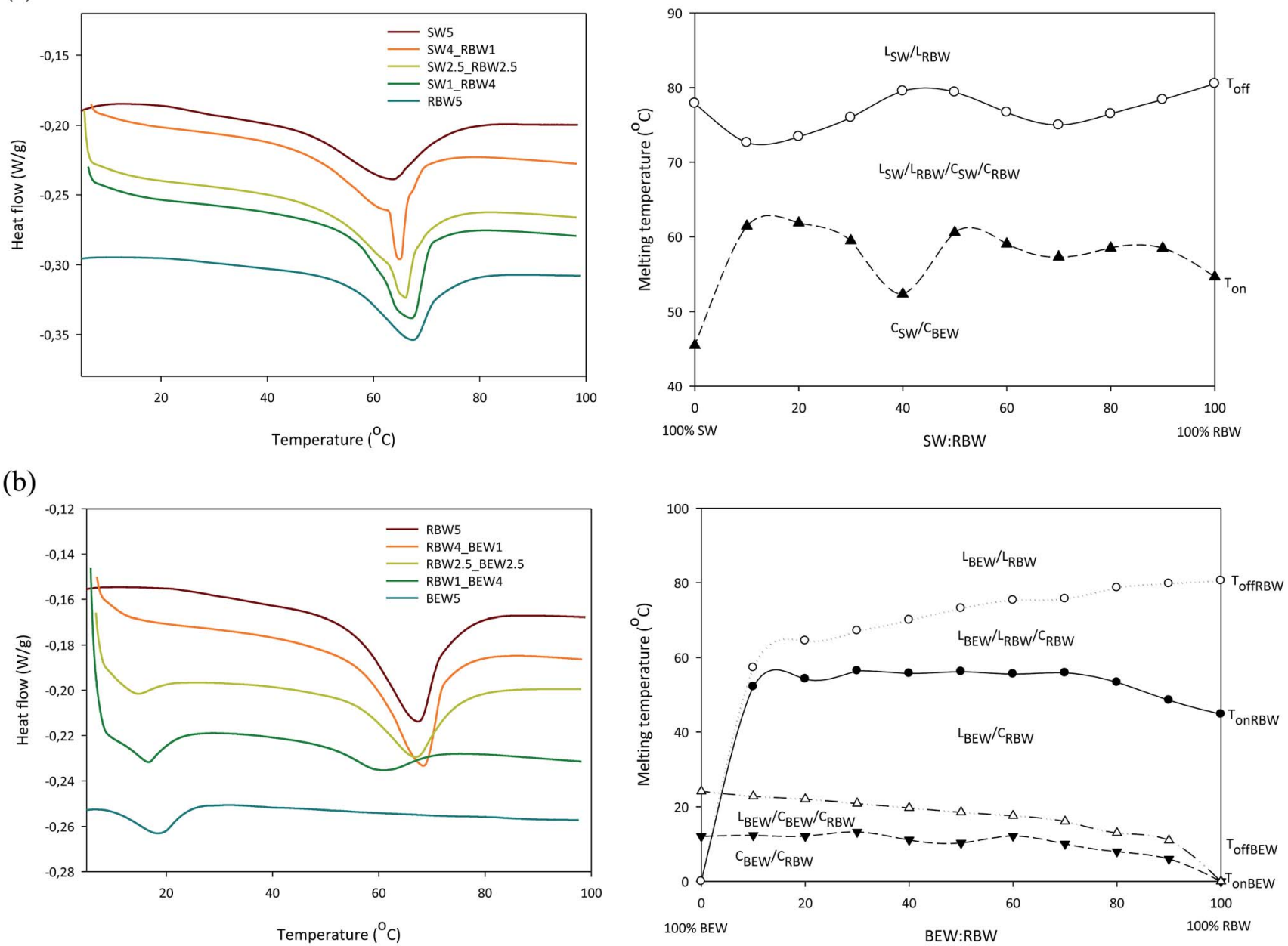

(c)
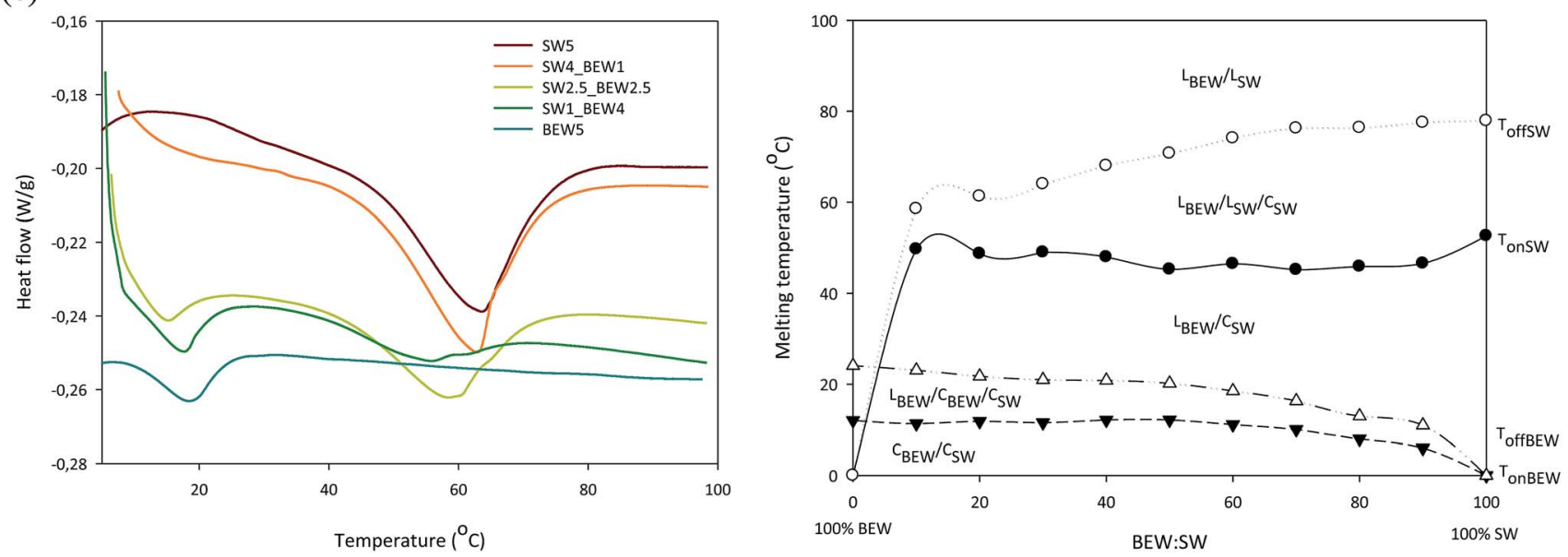

Fig. 1 Melting curves (left) and pseudo-phase diagrams (right) of oleogels stabilized with 5\% w/w SW : RBW (a); $5 \%$ w/w BEW : RBW (b) and 5\% w/ w BEW : SW (c).

sunflower oil and hazelnut oil. Also in these oils, RBW was not able to form a strong gel, therefore the authors assume that the bad gelation behavior of RBW is due to the chemical composition of the RBW. For the SW : RBW combination no increase in gel strength could be observed during the ageing period since $G^{\prime}$ remained constant throughout these $30 \mathrm{~min}$.
Fig. 2(b) and (c) demonstrate the sequential gelling of the high- and low-melting wax during cooling. Upon combining SW and BEW, the presence of SW initially results in a significant increase in $G^{\prime}$ during the cooling step. BEW only gels during the ageing step and further strengthens the crystal network already 

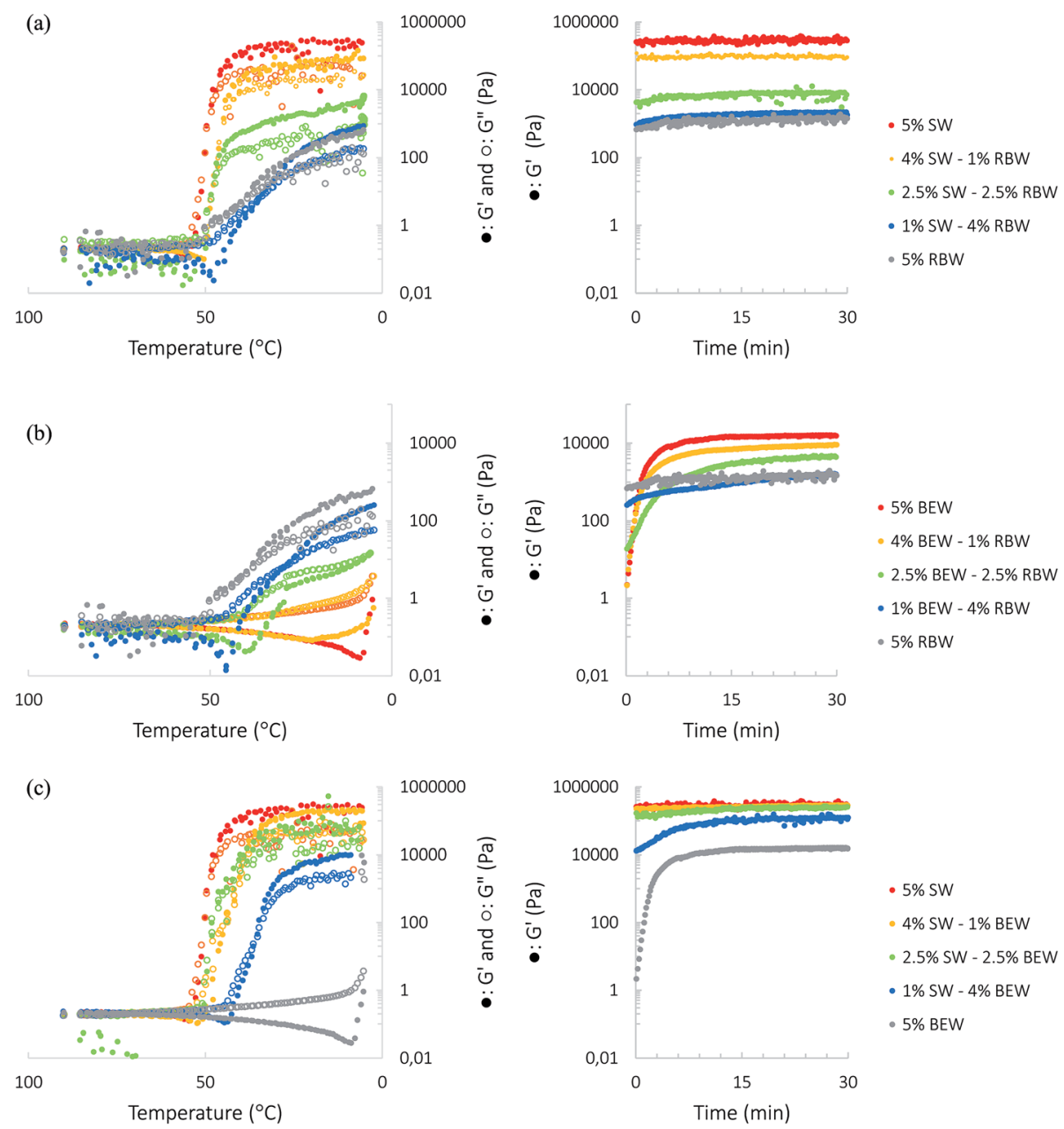

Fig. 2 Temperature sweeps (left) and subsequent time sweeps (right) of the 5\% SW : RBW oleogel (a), the 5\% SW : BEW oleogel (b) and the 5\% BEW : RBW oleogel (c).

formed by SW. Also when combining RBW with BEW, the BEW strengthens the network during ageing.

During cooling from 90 to $5{ }^{\circ} \mathrm{C}$, possibly there is insufficient undercooling to have crystallization of BEW $\left(T_{\text {c,peak }}(4 \% \mathrm{BEW})=\right.$ $7.4 \pm 0.2{ }^{\circ} \mathrm{C}$ and $\left.T_{\text {c,peak }}(2.5 \% \mathrm{BEW})=2.7 \pm 0.2^{\circ} \mathrm{C}\right)$ which is why the full crystallization of BEW only occurs during a storage period at $5{ }^{\circ} \mathrm{C}$. Furthermore, BEW contains slow crystallizing components (DAGs and TAGs), explaining the continuous crystallization during storage period.

To investigate whether the presence of SW and RBW crystals act as templates for BEW crystallization (heterogeneous nucleation), the crystallization temperatures $\left(T_{\mathrm{c}, \text { peak }}\right)$ of the mono wax-based oleogels and of the bi-wax-based oleogels were investigated (Table S1 $\dagger$ ). Sato (2001) stated that the chain length structure plays critical roles in the mixing-phase behavior of different types of the TAGs in solid phases. ${ }^{25}$ As determined by Doan et al. (2017) RBW and SW consist mainly out of long chain wax esters with a fatty acid moiety between $\mathrm{C}_{20}$ and $\mathrm{C}_{24}$ and a fatty alcohol moiety between $\mathrm{C}_{24}$ and $\mathrm{C}_{28} \cdot{ }^{12}$ BEW consists primarily out of fatty acids with a chain length of $\mathrm{C}_{16}$ and $\mathrm{C}_{18}$. Because of the significant difference in chain length, it is very unlikely that mixed crystals are formed. According to Smith, Bhaggan, Talbot, \& van Malssen (2011), it is even unlikely that the pre-formed SW and RBW crystals have a nucleation effect on the BEW crystallization because the requirement for a first stage crystal to influence second stage nucleation is that there should be sufficient similarity between the crystallizing species. ${ }^{26}$ From Table S1 $\uparrow$ can be deduced that the presence of RBW during cooling indeed did not influence the crystallization behavior of $\operatorname{BEW}\left(T_{\text {c,peak,low }}(4 \% \mathrm{BEW})=7.39 \pm 0.23{ }^{\circ} \mathrm{C}\right.$ and $T_{\text {c,peak,low }}(4 \%$ BEW : $1 \% \mathrm{RBW})=7.40 \pm 0.68^{\circ} \mathrm{C}$ ). SW did seem to influence the crystallization of BEW since $T_{\text {c,peak,low }}(4 \% \mathrm{BEW}: 1 \% \mathrm{SW})=6.22$ $\pm 0.02{ }^{\circ} \mathrm{C}$. In the presence of $1 \% \mathrm{SW}$, the crystallization of BEW appears to be delayed. For the $2.5 \% \mathrm{BEW}: 2.5 \% \mathrm{SW}$, there is no effect of the pre-existing crystals on the crystallization of BEW.

\section{Response of the binary wax-based oleogels to small and large deformations}

Oscillatory rheological behavior. Oscillatory rheological measurements allow the classification of samples into strong gels $\left(G^{\prime \prime} / G^{\prime} \leq 0.1\right)$, weak gels $\left(0.1<G^{\prime \prime} / G^{\prime}<1\right)$ and viscous sols $\left(G^{\prime \prime} / G^{\prime} \geq 1\right)$ because $G^{\prime}$ and $G^{\prime \prime}$ give an indication of the solid-like 
behavior and the liquid-like behavior, respectively. For gels, the elastic component $\left(G^{\prime}\right)$ dominates over the viscous component $\left(G^{\prime \prime}\right)$ at small applied oscillation stresses and attains a plateau in the linear-visco-elastic region (LVR). Within this LVR, the gel strength of a sample can be judged based on three factors: the difference between $G^{\prime}$ and $G^{\prime \prime}$, the average value of $G^{\prime}$ in the LVR $\left(G_{\text {LVR }}^{\prime}\right)$, and the length of the linear region of the curve. ${ }^{27}$ By convention the end of the LVR is the first point where $G^{\prime}$ varies by $10 \%$ of the $G_{\text {LVR }}^{\prime}$ value. The corresponding stress at this point is called the critical stress. Beyond the critical point, the samples undergo permanent deformation and the gel is transformed to a viscous sol at the cross-over point (the point where $G^{\prime}=G^{\prime \prime}$, called "oscillation yield stress"). A higher cross-over point is therefore also indicative of a stronger gel and a more stable network.

Amplitude sweep tests were performed at a frequency of $1 \mathrm{~Hz}$ and temperature of $5{ }^{\circ} \mathrm{C}$. Fig. 3 shows the plots of the oscillatory amplitude (left) and frequency (right) sweeps on the bi-waxbased oleogels at a total wax concentration of $5 \% \mathrm{w} / \mathrm{w}$. All samples reveal gel-like behavior with $G^{\prime}>G^{\prime \prime}$. For the SW : RBW combination, no sample is considered a strong gel since $G^{\prime \prime} / G^{\prime}$ is always larger than 0.1. However, the samples with a higher SW proportion do have a higher elastic modulus, indicating that SW crystallization results in better crystal network formation and better gelation of the oil. To investigate whether the addition of the weak gelator RBW has an actual structure weakening effect on the SW network, the oscillatory behavior of the $4 \%$ SW : $1 \%$ RBW was compared to that of $4 \%$ SW (Fig. S1 $\dagger$ ). Adding RBW effectively weakens the crystal network required for gelling and the observed effect was not solely due to a decrease in SW concentration.

The SW : BEW combination reveals some intriguing rheological results. The combinations $4 \%$ SW : 1\% BEW and 2.5\% SW : $2.5 \%$ BEW have a higher $G_{\text {LVR }}^{\prime}$, a higher critical stress and a higher cross-over point than the mono-wax oleogels $(5 \% \mathrm{BEW}$ and $5 \% \mathrm{SW}$ ), which is indicative of synergistic interactions between the two waxes. Interestingly, the higher the proportion of BEW is, the smaller the $G^{\prime \prime} / G^{\prime}$ ratio becomes. The $G^{\prime \prime} / G^{\prime}$ ratio (a)

(b)
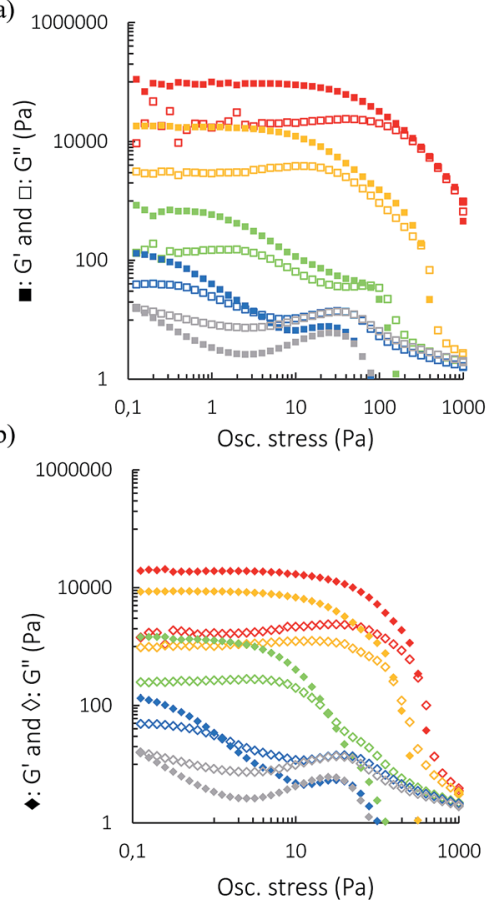

(c)

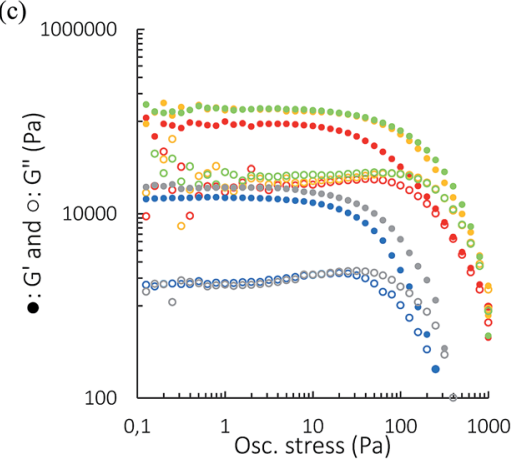

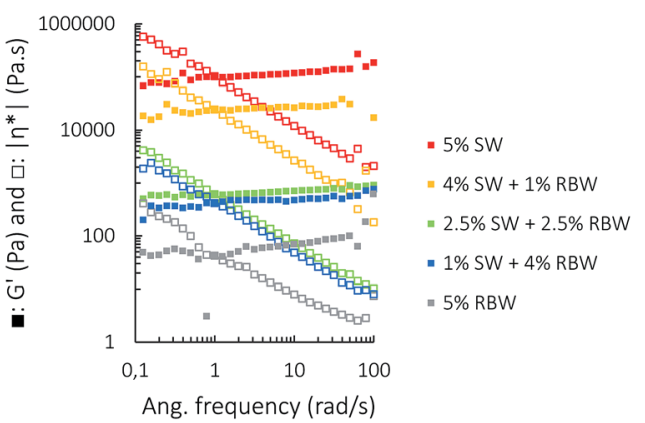
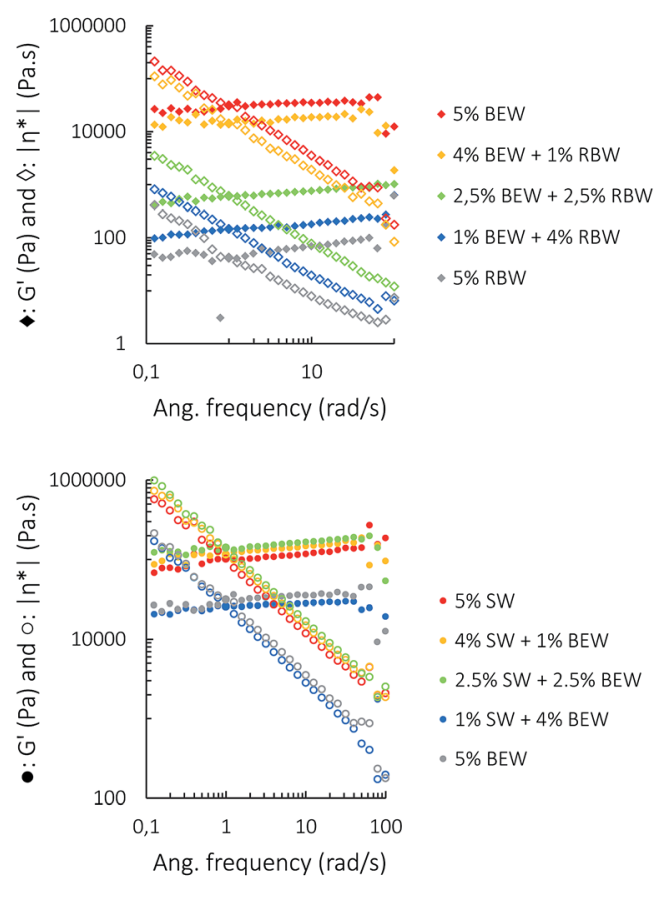

Fig. 3 Amplitude sweeps (left), frequency sweeps (right) for 5\% w/w bi-wax-based oleogels of SW : RBW (a), RBW : BEW (b) and SW : BEW (c). 
is smaller than 1 for the $5 \% \mathrm{w} / \mathrm{w}$ BEW oleogel, demonstrating that BEW oleogel solid-like properties strongly dominate over the liquid-like properties although it has a lower $G_{\text {LVR }}^{\prime}$ than SW oleogel. Furthermore, the LVR of BEW is longer than that of SW indicating that it is less brittle than the SW-oleogel. Yet the $5 \% \mathrm{~W} /$ w SW-oleogel has a higher cross-over point (where $G^{\prime}=G^{\prime \prime}$ ) than the BEW-oleogel, suggesting a better stability of SW-oleogels under the applied stress.

The same effect of BEW addition can be seen in the BEW : RBW combination. RBW is a very poor oleogelator in RBO which can be seen from the short LVR, the very low $G_{\text {LVR }}^{\prime}$ and the low cross-over point and the presence of RBW crystals seem to interfere with the formation of a good BEW crystal network, as can be seen from Fig. S2. $\dagger$

Frequency sweeps were executed to investigate the timedependent deformation of the oleogels. A stress value within the LVR was chosen and the angular frequency was increased from 0.1 to $100 \mathrm{rad} \mathrm{s}^{-1}$ while measuring the change in $G^{\prime}$ and $G^{\prime \prime}$. For all the combinations and for 5\% SW and 5\% BEW, the $G^{\prime}$ curve was mostly linear and inversely proportional to $\left|\eta^{*}\right|$ with the angular frequency, demonstrating that these gels had a good tolerance to the rate of deformation. The slight positive slope of the $G^{\prime}$ curve is characteristic for soft gels. ${ }^{27}$ The frequency sweeps confirm the synergistic interaction between SW and BEW at the combinations $4 \%$ SW : 1\% BEW and 2.5\% SW $: 2.5 \%$ BEW. The $G^{\prime}$ curve of the $5 \% \mathrm{w} / \mathrm{w}$ RBW oleogel was not linear, which again confirms the formation of a very weak RBW gel network.

Mechanical properties. The hardness of a wax-based oleogel is one of the most important aspects in determining the potential applicability of the oleogel in actual industrial products (foodstuffs, cosmetics or pharmaceutical products). The function of the wax is to give structure to the gel and keep it solid, therefore, the hardness of the oleogel has to be sufficiently high. ${ }^{28}$ Fig. 4 (top) confirms the results obtained from the rheological experiments. The replacement of a fraction of SW with RBW significantly decreases the hardness of the oleogel.

For the combinations of BEW with SW or RBW, very different results were obtained as compared to the SW : RBW combination. Surprisingly, the $4 \%$ BEW : $1 \% \quad$ RBW and $3.5 \%$ BEW : $1.5 \%$ RBW combinations show a significantly higher hardness than $5 \% \mathrm{BEW}$, which is not in accordance with the rheological results (amplitude and frequency sweeps). Narine and Marangoni (1999) state that there should be a direct relationship between the elastic modulus $G^{\prime}$ and the hardness index, which is not the case here. The explanation for this discordance can be the difference in storage time of the samples. The rheological measurements were done after 24 to $48 \mathrm{~h}$ storage at $5{ }^{\circ} \mathrm{C}$, while the hardness measurements presented here were done after 7 days of storage at $5{ }^{\circ} \mathrm{C}$. As discussed by Doan et al. (2016) there are polymorphic transitions within the BEW crystal network, which further reinforce the network after storage. ${ }^{30}$ In that case, the loose RBW crystals could act as a backbone for the recrystallization of the BEW, comparable to the SW crystals in the SW : BEW combination. The hardness measurements done after $48 \mathrm{~h}$ and after 2 weeks
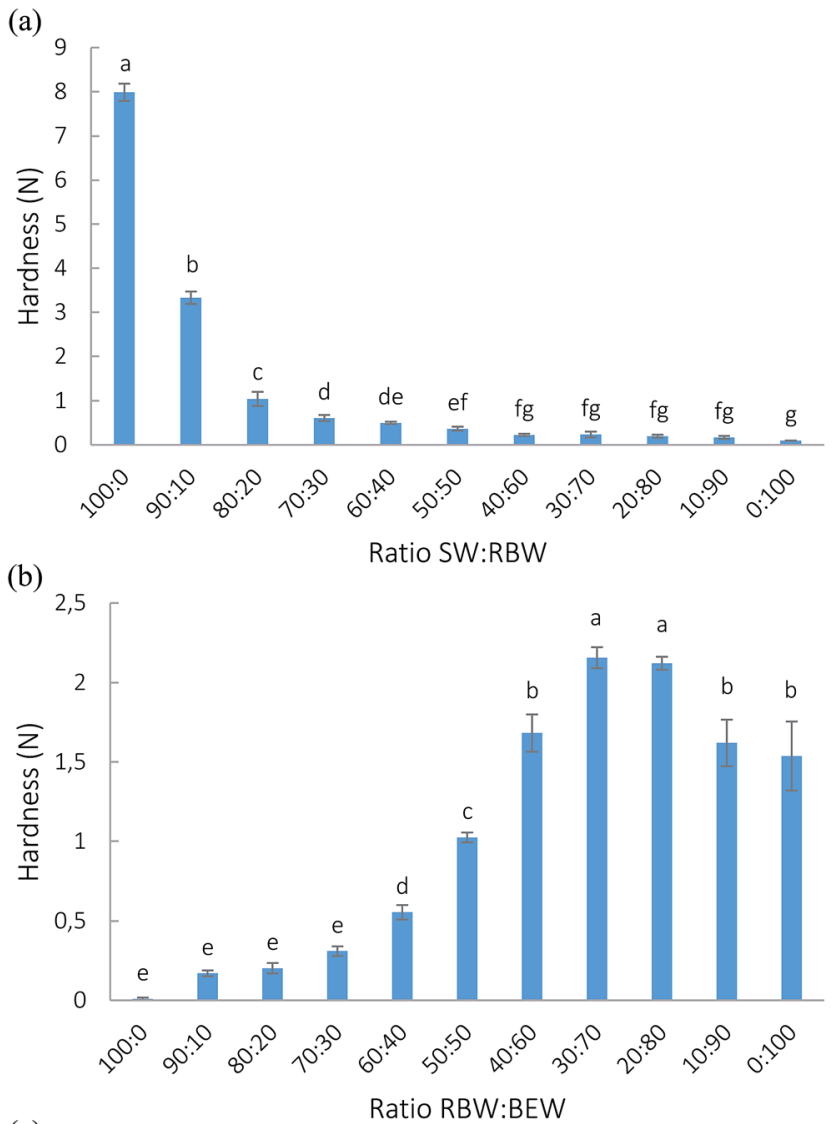

(c)

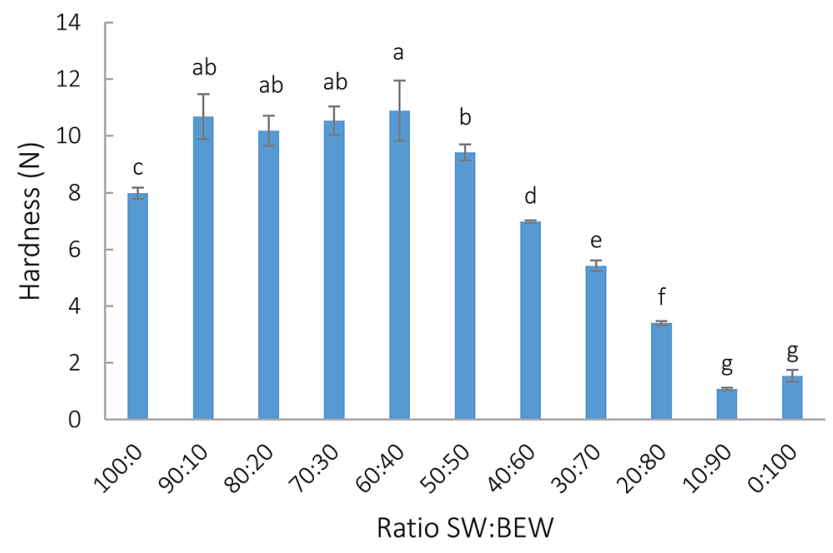

Fig. 4 Hardness of the $5 \% \mathrm{w} / \mathrm{w}$ bi-wax-based oleogels of SW : RBW (a), RBW : BEW (b) and SW : BEW (c).

indeed confirm an increase in hardness with time of the samples containing BEW (Fig. S3†). For the BEW : RBW combination, a rather high concentration of $\mathrm{BEW}(>3 \% \mathrm{BEW})$ is required to observe these synergistic interactions.

The results of the texture analysis of the SW : BEW combination are congruent with the small oscillatory stress experiments (amplitude sweeps and frequency sweeps). If SW is able to form a solid network (concentrations higher than $2.5 \% \mathrm{SW}$ ), BEW can further reinforce the crystal network.

The phenomenon of increased gel strength at certain ratios of high melting wax and low melting wax can have various 
reasons. First, the presence of high-melting wax crystals could have a nucleation effect, pushing the crystallization of BEW. However, as discussed previously, no positive effect on the crystallization of BEW from the high-melting crystals could be observed. Secondly, for TAG systems, the rheology is directly related to the solid fat content, ${ }^{31}$ and Narine and Marangoni (1999) stated that the particle size and the morphology of the microstructural elements also directly influence the rheological properties. ${ }^{29}$ Also polymorphism has a profound effect on the response of crystallized systems to deformations. Schaink, et al. (2007) found synergistic interactions in a fatty acid : fatty alcohol mixture, which was attributed to the formation of mixed crystals that were distinguished in XRD measurements. ${ }^{32}$ In wax-based systems, the hardness can be related to certain polymorphic forms. ${ }^{28}$ Another effect occurring is sintering or the formation of solid crystal bridges between larger crystals. Johansson et al. (1995) stated that sintering occurs when a fat phase crystallizes with a melting point between that of the oil and the crystal, as is the case here. ${ }^{33}$ To find a structural explanation for the phenomena under discussion, solid fat content and XRD measurements were done.

\section{Crystallization behavior of the binary wax-based oleogels}

Solid fat content. In systems where crystallized TAGs play an important role as structurants, a higher SFC is generally related to higher rheological and textural values. ${ }^{31}$ In such systems, the solid component forms a three-dimensional network that traps the liquid component. The higher the amount of solids, the more solid-like behavior of the fat. Possibly, the synergistic interactions between the high and low melting waxes in terms of hardness can also be explained by an increased solid fat content. Fig. 5 displays the $\mathrm{SFC}$ at $5{ }^{\circ} \mathrm{C}$ for the different combinations at 3 ratios $(20: 80,50: 50,80: 20)$. Clearly, the SFC and hardness are not related for these systems. Based on the hardness and rheological measurements, a gradual increase from $5 \%$ RBW to $5 \%$ SW would be expected. However, the SFC for the RBW : SW combinations is lower than that of the pure $5 \% \mathrm{SW}$ or RBW oleogels. This is indicative of some hindrance in crystallization when both are combined. For the SW/ RBW : BEW combinations, also a gradual increase in hardness from RBW/SW to $5 \%$ BEW can be noticed. A synergistic effect in terms of crystalline mass cannot be discerned. Toro-Vazquez et al. (2007) and Doan et al. (2015) already found that the hardness and SFC are not related for wax-based systems but that the hardness depends mostly on the average size and shape of the solid particles and the three-dimensional organization of the solid phase. ${ }^{14,15}$

Powder X-ray diffraction. X-ray diffraction (XRD) measurements were used to investigate the molecular arrangements of the SW, RBW and BEW crystals if combined. Measurements were done in both the wide diffraction angle diffraction (WAXD) region for short-spacing runs and in the small diffraction angle diffraction (SAXD) region for long-spacing runs. WAXD provides information on the lateral packing and SAXS on the longitudinal packing of molecules in crystalline structures. A change in peak position is indicative of a change in crystal polymorphic

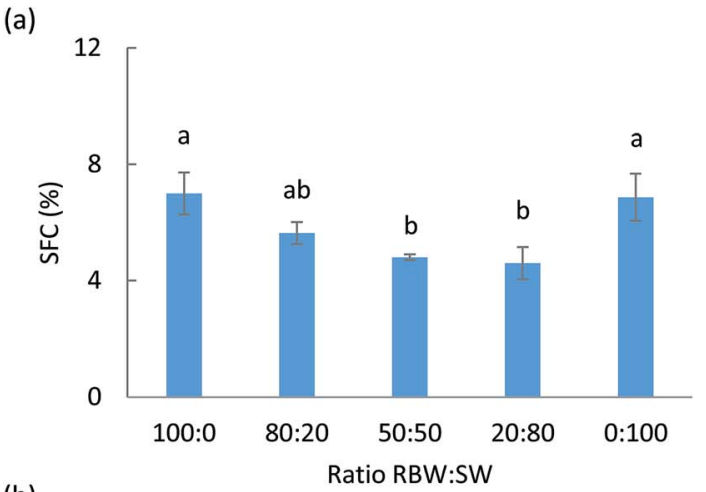

(b)

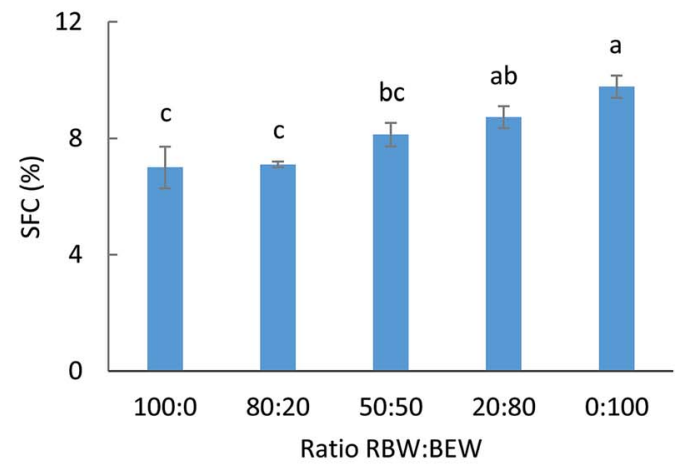

(c)

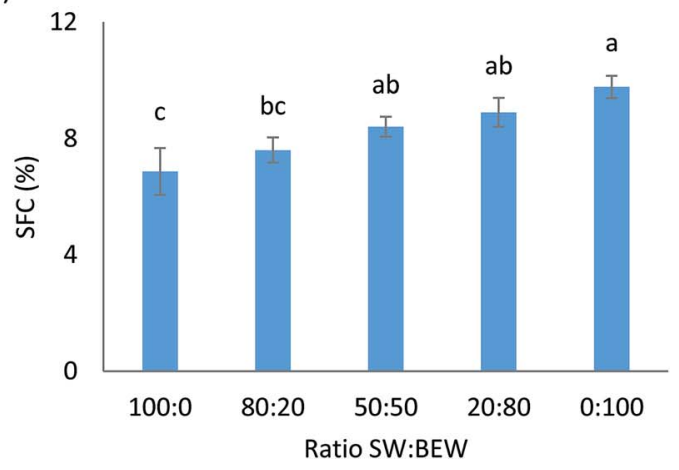

Fig. 5 Solid fat content measured at $5{ }^{\circ} \mathrm{C}$ of the three wax-based oleogels at a total wax concentration of $5 \%$ : RBW : SW (a), RBW : BEW (b) and SW : BEW (c).

behavior which could possible explain the observed variation in the hardness. Braipson-Danthine and Deroanne (2004) studied binary blends of fats and were able to relate the variation in hardness to a change in polymorphic form. ${ }^{34}$ Schaink, et al. (2007) found synergistic interaction between a fatty acid and a fatty alcohol, which were explained by a change in the layer thickness observed in the wide-angle diffraction range. The systems studied here have a very low amount of crystal matter, which results in low peak intensities. Furthermore, as stated previously, waxes consist of a variety in molecules (hydrocarbons, wax esters, fatty acids, and fatty alcohols), which makes it challenging to extrapolate XRD data on regular TAG-structured fats. Therefore, we will mainly focus on the occurrence of new peaks or peak shifts upon combining two waxes. Fig. 6 shows the small angle (left) and wide angle (right) X-ray diffractograms 
of the bi-wax-based oleogels at $5{ }^{\circ} \mathrm{C}$. For the RBW : SW combination (Fig. 6a), the presence of two separated intensive and narrow peaks at $d$-spacing of $0.413 \mathrm{~nm}$ and $0.373 \mathrm{~nm}(d=n \times$ wavelength $/ 2 \times \sin ($ theta) $)$ are observed in the wide angle region (right).

These $d$-spacing values are indicative of the orthorhombic sub-cell structure or $\beta^{\prime}$ morphology for both RBW and SW. ${ }^{25}$ The measurements done in the SAXS region (long spacings) are associated with layer staggering which is in its turn is determined by chain length, chain tilt and number of chains per layer. ${ }^{32}$ The SAXS measurements show no change in $d$-spacing of the bi-wax-based system compared to that of the mono-wax based systems, which means the layer staggering does not change upon combing the two waxes, excluding the possibility of mixed crystal formation.

The combinations of a high melting wax (SW or RBW) with the BEW (Fig. 6b and c) reveal several peaks in the WAXD region. The peaks at $d$-spacing of $0.413 \mathrm{~nm}$ and $0.373 \mathrm{~nm}$ allow the identification of the orthorhombic sub-cell structure $\beta^{\prime}$, which are associated with the high-melting wax. The peak at a $d$ spacing of $0.388 \mathrm{~nm}$ could be indicative of the $\gamma$ polymorphic form, especially taking into account previous work revealing a clear peak at $d$-spacing $0.473 \mathrm{~nm}$ in the BEW diffractogram after 3 weeks of storage. ${ }^{30,35}$ The combinations of RBW : BEW and SW : BEW show the presence of both the $\beta^{\prime}$ polymorph (from the high melting wax) and the $\gamma$ polymorph (from the (a)

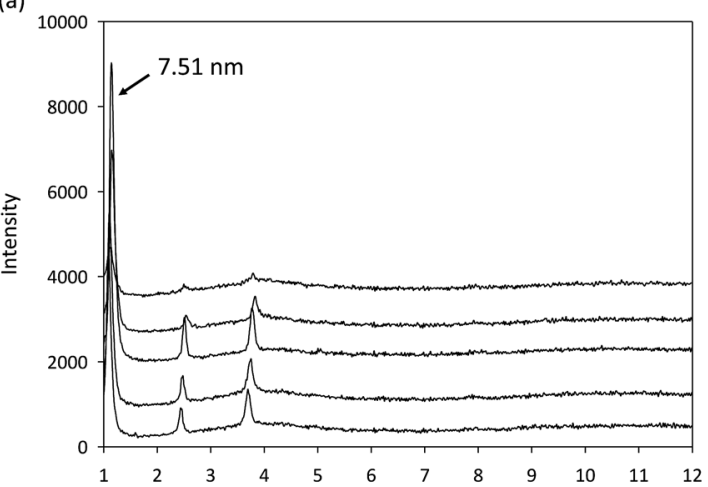

(b)
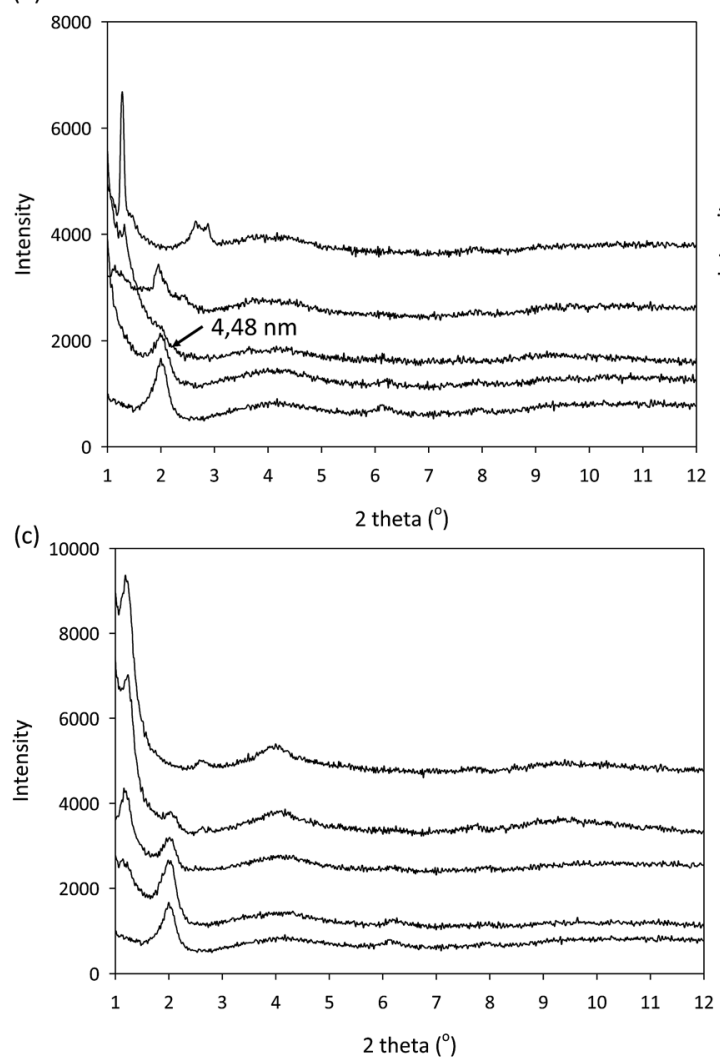
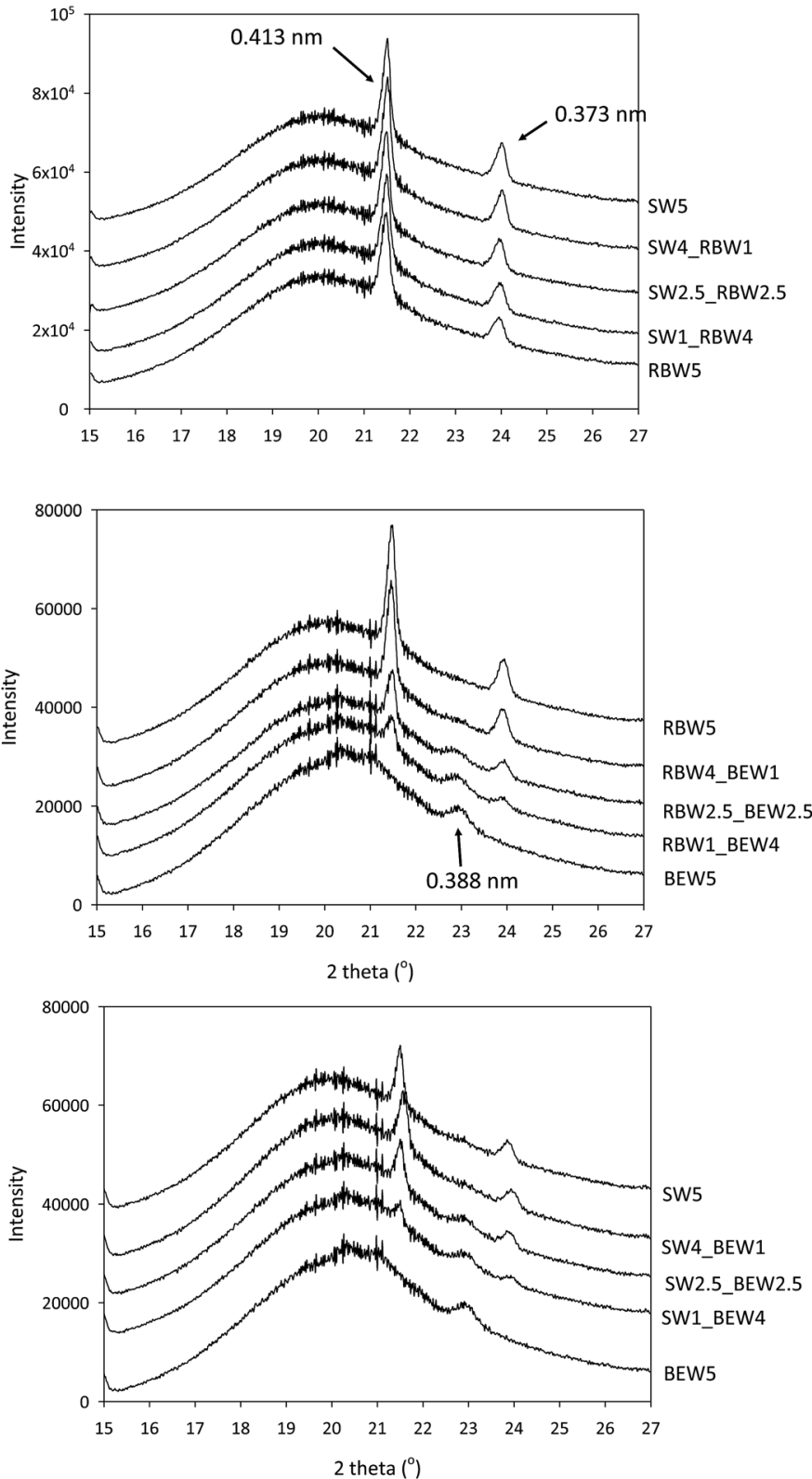

Fig. 6 Small angle (left) and wide angle (right) X-ray diffractograms of wax-based oleogels at $5{ }^{\circ} \mathrm{C}$, stabilized with a combination of $5 \%$ w/W RBW : SW (a), RBW : BEW (b) and SW : BEW (c). 
BEW). In the SAXS region one can again very clearly distinguish which peaks originate from BEW and which from the high melting waxes. RBW, SW and BEW show the appearance of one intense and strong reflection (001) peak, followed by the intensity decrease of other higher-order peaks. The (001) reflection peak of SW and RBW appears at $75.1 \mathrm{~nm}$ which corresponds to a triple chain length stacking $(3 L)$, while the (001) peak of BEW is at $44.8 \mathrm{~nm}$, indicative of a $2 L$ packing.

No shift in peak position or new polymorphic forms could be observed when the waxes were combined. The XRD measurements therefore established that combining these waxes results in co-existing crystals rather than mixed crystals. Polymorphic changes do not explain the variation in hardness previously observed.

Crystal morphology. Fig. 7 depicts the micrographs of the $5 \%$ w/w mono-wax oleogels with PLM (top) and cryo-SEM (below). From the PLM images, RBW appears to crystallize as large dendritic crystals in RBO which do not form a tight network and can therefore not contain large amounts of liquid oil in its cavities. This explains why RBW is considered to be a weak gelator in RBO and thus higher concentrations of RBW are required to form an oleogel in RBO. ${ }^{\mathbf{1 4}}$ The SW and BEW crystals appear to be needle-like in morphology, with the SWcrystals slightly bigger in size. ${ }^{7,8}$ However, a recent study by Blake and Marangoni (2015) reports on the occurrence of platelet-like crystals for RBW and SW in peanut oil, which was visualized with cryo-SEM. ${ }^{36}$ They suggest to use cryo-SEM imaging as the main imaging tool for visualization of crystal networks and to consider surface area instead of wax crystal morphology as the main factor determining the oil structuring capacity of a wax. In the cryo-SEM images in Fig. 7, the $5 \% \mathrm{w} / \mathrm{w}$ RBW and $5 \% \mathrm{w} / \mathrm{w}$ SW oleogels contain platelet crystals.
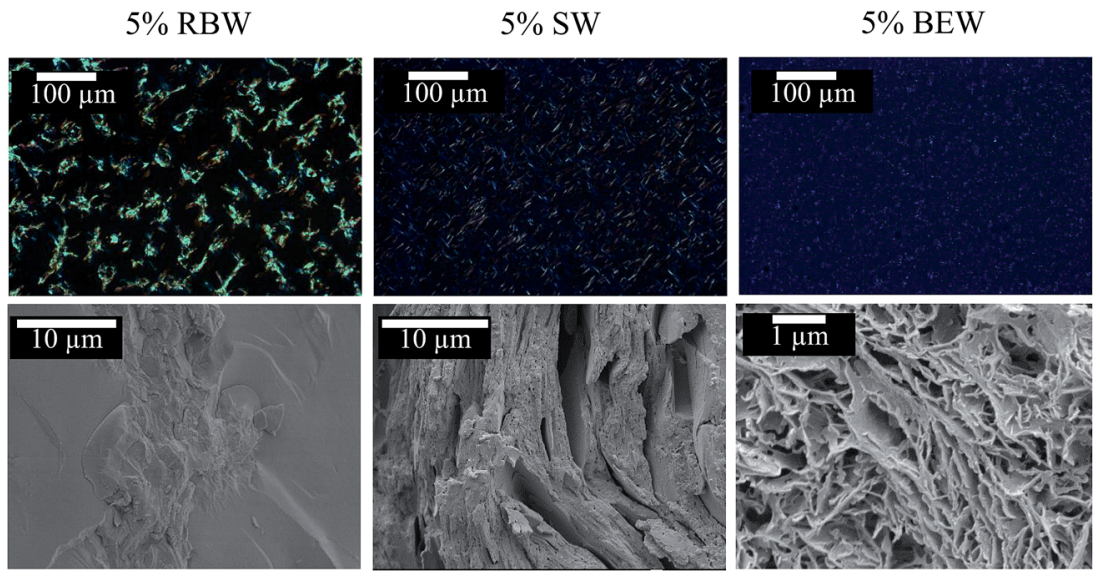

Fig. 7 Micrographs of the crystal structure in a 5\% SW (left), 5\% RBW (middle) and 5\% BEW oleogel (right) with PLM (top); and with cryo-SEM (bottom).

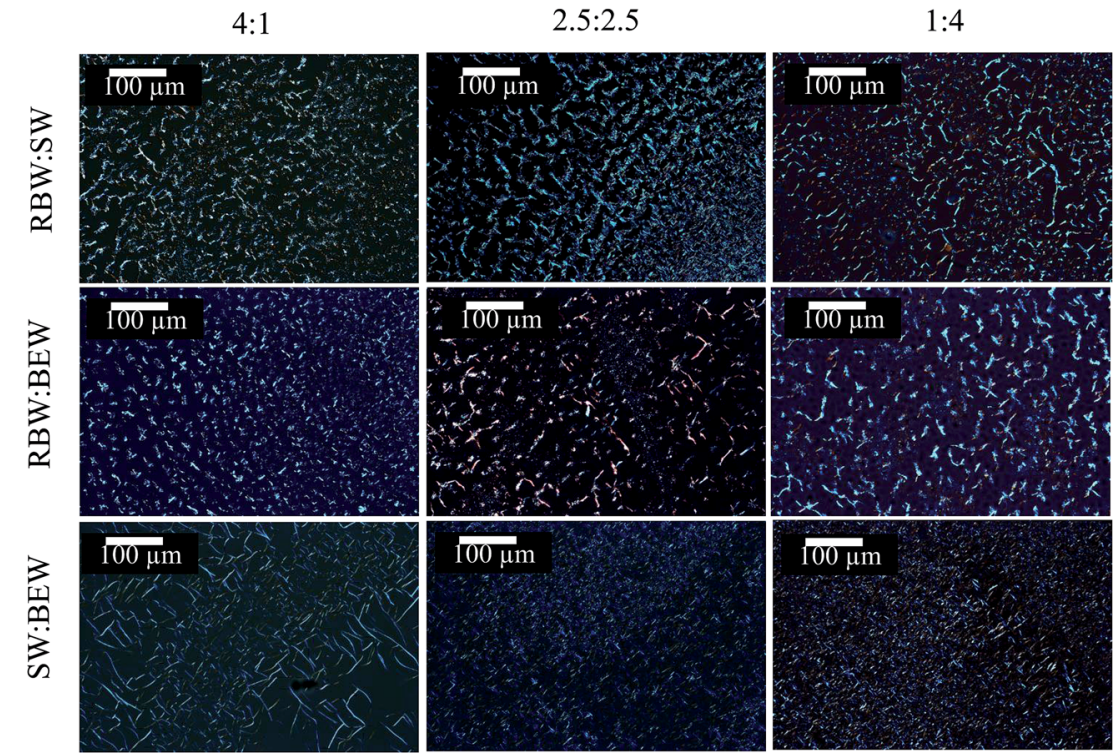

Fig. 8 Polarized light microscopy images of wax-based oleogels, stabilized with a combination of 5\% w/w RBW : SW (top); 5\% w/w RBW : BEW (middle) and 5\% w/w SW : BEW (below), in various ratios (4:1, left; $2.5: 2.5$, middle; $1: 4$, right). 
However, the crystal organization is very different. The platelets of the RBW appear to be randomly organized in clusters, which are not interconnected, while the SW platelets are piled upon each other and form a network. To support this statement, a more zoomed in and zoomed out cryo-SEM image of the RBWoleogel is shown in Fig. S4. $\dagger$ The BEW crystals do not appear as platelets, but more as sponge-like structure. Cryo-SEM imaging therefore confirms the importance of the surface area on the oil binding and gelation capacity of the three different wax-based oleogels since RBW crystal networks clearly have a lower surface area than the networks of SW and BEW, which are considered to be good oleogelators.

The appearance of crystalline particles during cooling of the $5 \% \mathrm{w} / \mathrm{w}$ bi-wax-based oleogels was elucidated only under polarized light microscopy, because it appeared to be challenging to discern the different crystal morphologies under cryo-SEM (Fig. 8). The oleogel samples were placed on a microscope slide, heated to $90{ }^{\circ} \mathrm{C}$ and cooled down at a rate of $5{ }^{\circ} \mathrm{C}$ $\min ^{-1}$ to $5{ }^{\circ} \mathrm{C}$, to allow visualization of the sequential crystallization of the waxes (Fig. 5). For the RBW : SW combination, initially SW crystals appeared and a few seconds later also RBW could be seen, confirming the simultaneous crystallization of the two crystal types. No mixed crystals could be observed.

For the combination of a high and a low-melting wax, first the high-melting crystals appeared (SW and RBW), followed by the low-melting wax (BEW). In the micrographs, the different crystal morphologies can be identified. The RBW and SW crystals are big compared to the small BEW crystals. The BEW crystals filled up the voids left where no SW or RBW crystals had appeared. The crystal structures change with the proportion of wax changes in the binary blends. A higher proportion of a certain wax in the melt, also results in a higher proportion of the respective crystal morphology in the solidified sample.

We hypothesize that the increase in hardness can be explained by a process called sintering. Sintering involves involves the formation of solid bridges within the fat crystal networks. ${ }^{33}$ Ojijo et al. (2004) found an increase in hardness during storage in a system containing monoacylglycerols structuring olive oil and attributed this effect to sintering. ${ }^{37}$ In our opinion, a similar process is occurring upon combining high and low melting waxes. During cooling, initially the high melting waxes crystallize. Subsequently, the low melting waxes are able to form solid bridges in between the already present high melting waxes, hereby increasing the hardness. For the SW : BEW system, only a small amount of BEW was required to introduce sintering because SW already forms a dense crystal network (Fig. 4). The synergistic interaction was therefore found at a ratio of $1 \% \mathrm{BEW}: 4 \% \mathrm{SW}$. In the RBW : BEW system, a higher amount of BEW was required because RBW forms a loose crystal network structure. The synergistic interaction could therefore only be detected at a ratio of $4 \%$ BEW : $1 \%$ RBW.

\section{Conclusion}

Oleogels were prepared with three binary wax combinations: SW and RBW, RBW and BEW and SW and BEW. Based on the results from DSC analysis, temperature sweeps and polarized microscopy, it could be concluded that BEW, a low melting wax, combined with SW or RBW, high melting waxes, present sequential crystallization and melting behavior. RBW and SW crystallize and melt simultaneously, but no mixed crystals were formed. Interestingly, the BEW crystals were able to reinforce the network structure formed by the high-melting waxes by forming solid crystal bridges between the pre-existing SW and RBW crystals (sintering). This effect was detected with small angle oscillatory stress experiments and texture analysis. This research clearly presents the possibility of combining high- and low melting substances in tailoring the physical properties of the oleogel. Here, BEW, SW and RBW were used as example materials, but also other waxes with differences in melting behavior could be used and even other crystallizing materials (e.g. mono- and di-glycerides) could be combined and enhanced structuring can be obtained.

\section{Acknowledgements}

This research was made possible thanks to the BOF (Special Research Fund) of Ghent University and the Erasmus Mundus scholarship (Lotus III Project). Vandemoortele is recognized for its financial help in the acquisition of the Leica polarized light microscope and the scientific input. Hercules foundation is recognized for its financial support in the acquisition of the scanning electron microscope JEOL JSM-7100F equipped with cryo-transfer system Quorum PP3000T (grant number AUGE-09029).

\section{Notes and references}

1 R. P. Mensink and M. B. Katan, Effect of Dietary trans Fatty Acids on High-Density and Low-Density Lipoprotein Cholesterol Levels in Healthy Subjects, N. Engl. J. Med., 1990, 323(7), 439-445, DOI: 10.1056/nejm199008163230703.

2 D. Mozaffarian, M. B. Katan, A. Ascherio, M. J. Stampfer and W. C. Willett, Trans fatty acids and cardiovascular disease, $N$. Engl. J. Med., 2006, 354, 1601-1613.

3 R. Chowdhury, S. Warnakula, S. Kunutsor, F. Crowe, H. A. Ward, L. Johnson, et al., Association of Dietary, Circulating, and Supplement Fatty Acids With Coronary Risk: A Systematic Review and Meta-analysis, Ann. Intern. Med., 2014, 160(6), 398-406.

4 P. Terech and R. G. Weiss, Low Molecular Mass Gelators of Organic Liquids and the Properties of Their Gels, Chem. Rev., 1997, 97(97), 3133-3160.

5 E. D. Co and A. G. Marangoni, Organogels: An Alternative Edible Oil-Structuring Method, J. Am. Oil Chem. Soc., 2012, 89(5), 749-780.

6 A. R. Patel, Alternative Routes to Oil Structuring [Internet], Springer, 2015, pp. 15-28, available from: http:// link.springer.com/10.1007/978-3-319-19138-6.

7 A. I. Blake, E. D. Co and A. G. Marangoni, Structure and physical properties of plant wax crystal networks and their relationship to oil binding capacity, JAOCS, J. Am. Oil Chem. Soc., 2014, 91(6), 885-903. 
8 H. S. Hwang, S. Kim, M. Singh, J. K. Winkler-Moser and S. X. Liu, Organogel formation of soybean oil with waxes, JAOCS, J. Am. Oil Chem. Soc., 2012, 89(4), 639-647.

9 L. S. K. Dassanayake, D. R. Kodali and S. Ueno, Formation of oleogels based on edible lipid materials, Curr. Opin. Colloid Interface Sci., 2011, 16(5), 432-439.

10 T. C. S. Kanya, L. J. Rao and M. C. S. Sastry, Characterization of wax esters, free fatty alcohols and free fatty acids of crude wax from sunflower seed oil refineries, Food Chem., 2007, 101(4), 1552-1557.

11 S. R. Vali, Y. H. Ju, T. N. B. Kaimal and Y. T. Chern, A process for the preparation of food-grade rice bran wax and the determination of its composition, JAOCS, J. Am. Oil Chem. Soc., 2005, 82(1), 57-64.

12 C. D. Doan, C. M. To, M. De Vrieze, F. Lynen, S. Danthine, A. Brown, et al., Chemical profiling of the major components in natural waxes to elucidate their role in liquid oil structuring, Food Chem., 2017, 214, 717-725.

13 A. R. Patel, M. Babaahmadi, A. Lesaffer and K. Dewettinck, Rheological Profiling of Organogels Prepared at Critical Gelling Concentrations of Natural Waxes in a Triacylglycerol Solvent, J. Agric. Food Chem., 2015, 63(19), 4862-4869.

14 C. D. Doan, D. Van de Walle, K. Dewettinck and A. R. Patel, Evaluating the Oil-Gelling Properties of Natural Waxes in Rice Bran Oil: Rheological, Thermal, and Microstructural Study, J. Am. Oil Chem. Soc., 2015, 92(6), 801-811, DOI: 10.1007/s11746-015-2645-0.

15 J. F. Toro-Vazquez, J. A. Morales-Rueda, E. DibildoxAlvarado, M. Charó-Alonso, M. Alonzo-Macias and M. M. González-Chávez, Thermal and textural properties of organogels developed by candelilla wax in safflower oil, JAOCS, J. Am. Oil Chem. Soc., 2007, 84(11), 989-1000.

16 L. S. K. Dassanayake, D. R. Kodali, S. Ueno and K. Sato, Physical properties of rice bran wax in bulk and organogels, JAOCS, J. Am. Oil Chem. Soc., 2009, 86(12), 1163-1173.

17 E. Yilmaz and M. Öğütcü, Comparative Analysis of Olive Oil Organogels Containing Beeswax and Sunflower Wax with Breakfast Margarine, J. Food Sci., 2014, 79(9), E1732-E1738.

18 S. Martini, A. A. Carelli and J. Lee, Effect of the addition of waxes on the crystallization behavior of anhydrous milk fat, JAOCS, J. Am. Oil Chem. Soc., 2008, 85(12), 1097-1104.

19 S. Jana and S. Martini, Phase Behavior of Binary Blends of Four Different Waxes, J. Am. Oil Chem. Soc., 2016, 93(4), 543-554.

20 S. Jana and S. Martini, Physical characterization of crystalline networks formed by binary blends of waxes in soybean oil, Food Res. Int., 2016, 89, 245-253, DOI: 10.1016/j.foodres.2016.08.003.

21 F. Yu, S. H. Kim, N. S. Kim, J. H. Lee, D. H. Bae and K. T. Lee, Composition of solvent-fractionated rice bran oil, J. Food Lipids, 2006, 13(3), 286-297.

22 R. Zantl, L. Baicu, F. Artzner, I. Sprenger, G. Rapp and J. O. Rädler, Thermotropic Phase Behavior of Cationic
Lipid-DNA Complexes Compared to Binary Lipid Mixtures, J. Phys. Chem. B, 1999, 103(46), 10300-10310, DOI: 10.1021/ jp991596j.

23 C. Himawan, V. M. Starov and A. G. F. Stapley, Thermodynamic and kinetic aspects of fat crystallization, Adv. Colloid Interface Sci., 2006, 122(1-3), 3-33.

24 S. Martini, C. Y. Tan and S. Jana, Physical Characterization of Wax/Oil Crystalline Networks, J. Food Sci., 2015, 80(5), C989C997.

25 K. Sato, Crystallization behaviour of fats and lipidsa review, Chem. Eng. Sci., 2001, 56(7), 2255-2265.

26 K. W. Smith, K. Bhaggan, G. Talbot and K. F. van Malssen, Crystallization of Fats: Influence of Minor Components and Additives, J. Am. Oil Chem. Soc., 2011, 88(8), 1085-1101.

27 T. G. Mezger, The Rheology Handbook, Vincentz Network GmbH \& Co. KG, Hannover, 3rd edn, 2011, pp. 33-89.

28 T. Imai, K. Nakamura and M. Shibata, Relationship between the hardness of an oil-wax gel and the surface structure of the wax crystals, Colloids Surf., A, 2001, 194(1-3), 233-237.

29 S. S. Narine and A. G. Marangoni, Relating structure of fat crystal networks to mechanical properties: a review, Food Res. Int., 1999, 32(4), 227-248.

30 C. D. Doan, I. Tavernier, M. D. Bin Sintang, S. Danthine, D. Van de Walle, T. Rimaux, et al., Crystallization and Gelation Behavior of Low- and High Melting Waxes in Rice Bran Oil: A Case-Study on Berry Wax and Sunflower Wax, Food Biophys., 2017, 12(1), 97-108.

31 R. Campos, S. S. Narine and A. G. Marangoni, Effect of cooling rate on the structure and mechanical properties of milk fat and lard, Food Res. Int., 2002, 35, 971-981.

32 H. M. Schaink, K. F. van Malssen, S. Morgado-Alves, D. Kalnin and E. van der Linden, Crystal network for edible oil organogels: possibilities and limitations of the fatty acid and fatty alcohol systems, Food Res. Int., 2007, 40(9), 1185-1193.

33 D. Johansson and B. Bergenståhl, Sintering of fat crystal networks in oil during post-crystallization processes, J. Am. Oil Chem. Soc., 1995, 72(8), 911-920, DOI: 10.1007/ BF02542069.

34 S. Braipson-Danthine and C. Deroanne, Influence of SFC, microstructure and polymorphism on texture (hardness) of binary blends of fats involved in the preparation of industrial shortenings, Food Res. Int., 2004, 37(10), 941-948.

35 S. Ueno and A. Minato, Synchrotron Radiation X-ray Diffraction Study of Liquid Crystal Formation and Polymorphic Crystallization of SOS (sn-1,3-distearoyl-2oleoyl glycerol), J. Phys. Chem., 1997, 5647(1), 6847-6854.

36 A. I. Blake and A. G. Marangoni, Plant wax crystals display platelet-like morphology, Food Struct., 2015, 3, 30-34, DOI: 10.1016/j.foostr.2015.01.001.

37 N. K. O. Ojijo, E. Kesselman, V. Shuster, S. Eichler, S. Eger, I. Neeman, et al., Changes in microstructural, thermal, and rheological properties of olive oil/monoglyceride networks during storage, Food Res. Int., 2004, 37(4), 385-393. 